\title{
Combination of shower-front sampling and imaging in the Tunka Advanced International Gamma-ray and Cosmic ray Astrophysics (TAIGA) project
}

Maike Kunnas $^{* a}$, I. Astapov ${ }^{j}$, N. Barbashina ${ }^{j}$, S. Beregnev ${ }^{c}$, A. Bogdanov $^{g}$, D. Bogorodskii $^{d}$, V. Boreyko ${ }^{b}$, M. Brückner ${ }^{e}$, N. Budnev ${ }^{d}$, A. Chiavassa ${ }^{h}$, O. Chvalaev $^{d}$,

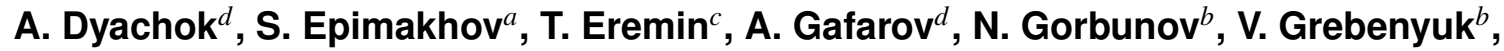

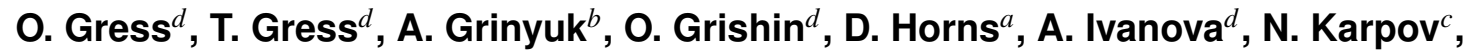
N. Kalmykov ${ }^{c}$, Y. Kazarina ${ }^{d}$, V. Kindin ${ }^{g}$, N. Kirichkov ${ }^{d}$, S. Kiryuhin ${ }^{d}$, R. Kokoulin ${ }^{g}$, K. Kompaniets $^{g}$, E. Konstantinov ${ }^{d}$, A. Korobchenko ${ }^{d}$, E. Korosteleva ${ }^{c}$, V. Kozhin ${ }^{c}$, L. Kuzmichev $^{c}$, V. Lenok ${ }^{d}$, B. Lubsandorzhiev ${ }^{k}$, N. Lubsandorzhiev ${ }^{c}$, R. Mirgazov $^{d}, \mathbf{R}$. Mirzoyan $^{i}{ }^{d}$, R. Monkhoev ${ }^{d}$, Müger $^{e} f$, R. Nachtigall ${ }^{a}$, A. Pakhorukov ${ }^{d}$, M. Panasyuk ${ }^{c}$, L. Pankov ${ }^{d}$, A. Perevalov ${ }^{d}$, A. Petrukhin ${ }^{g}$, V. Platonov ${ }^{d}$, V. Poleschuk ${ }^{d}$, M. Popescu ${ }^{l}$, E. Popova ${ }^{c}$, A. Porelli ${ }^{f}$, S. Porokhovoy ${ }^{b}$, E. Postnikov ${ }^{c}$ V. Prosin ${ }^{c}$, V. Ptuskin ${ }^{j}$, V. Romanov $^{b}$, G.I. Rubtsov ${ }^{k}$, E. Rybov ${ }^{d}$, V. Samoliga ${ }^{d}$, P. Satunin ${ }^{k}$, A. Saunkin ${ }^{d}$, V. Savinov $^{d}$, Yu. Semeney $^{d}$, B. Shaibonov(junior) ${ }^{k}$, A. Silaev $^{c}$, A. Silaev (junior) ${ }^{c}, \mathbf{A}$. Skurikhin $^{c}$, M. Slunecka $^{b}$, C. Spiering ${ }^{f}$, L. Sveshnikova ${ }^{c}$, V. Tabolenko ${ }^{d}$, A. Tkachenko $^{b}$, L. Tkachev ${ }^{b}$, M. Tluczykont ${ }^{a}$, A. Veslopopov ${ }^{d}$, E. Veslopopova ${ }^{d}$, D.

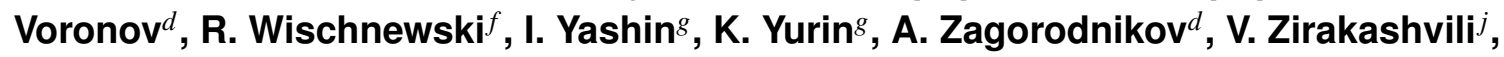
V. Zurbanov ${ }^{d}$

${ }^{a}$ Institut für Experimentalphysik, Luruper Chaussee 149, 22761 Hamburg, Germany

${ }^{b}$ Joint Institute for Nuclear Research, Joliot-Curie 6, 141980 Dubna, Moscow region, Russia

c Skobeltsyn institute for Nuclear Physics, Lomonosov Moscow State University, 1 Leninskie gory, 119991 Moscow, Russia

${ }^{d}$ Institute of Applied Physics, Irkutsk State University, Irkutsk, Russia

e Institute for Computer Science, Humboldt-University Berlin, Rudower Chaussee 25, 12489 Berlin, Germany

${ }^{f}$ DESY, Platanenallee 6, 15738 Zeuthen, Germany

${ }^{g}$ MEPhI (Moscow Engineering Physics Insitute), National Research Nuclear University, Moscow, Russia

${ }^{h}$ Dipartimento di Fisica Generale Universiteta di Torino and INFN, Torino, Italy

${ }^{i}$ Werner Heisenberg Institut, Föhringer Ring 6, 80805, München, Germany

${ }^{j}$ IZMIRAN, Troitsk, Moscow Region, Russia

${ }^{k}$ Institute for Nuclear Research of the Russian Academy of Sciences 60th October Anniversary st., 7a, 117312, Moscow, Russia

${ }^{l}$ Institute of Space Science, Bucharest, Romania

E-mail: maike.kunnas@desy.de 
For observation of extensive air showers (EAS) from gamma rays and cosmic rays, Cherenkov light sampling arrays (non-imaging technique or timing-arrays) provide good core position, energy, and angular resolution similar to imaging telescopes (IACTs) and yield good sensitivity at the highest energies due to the potentially large collection area. However, the gamma-hadron separation power of this method limits the sensitivity at the energy threshold.

In matters of gamma hadron separation and reconstruction, imaging air Cherenkov telescopes are the instrument of choice, but a stereoscopic view of a shower is needed for accurate geometrical reconstruction. This makes it costly to achieve sufficiently large effective areas needed for sensitive observations at and above $100 \mathrm{TeV}$.

A combination of these experimental approaches, using the respective strength of both techniques and compensating their weaknesses, could optimise the sensitivity.

The TAIGA project will for the first time combine a non-imaging array with small $\left(\mathrm{O}\left(10 \mathrm{~m}^{2}\right)\right.$ mirror) imaging telescopes. Here, we present the first hybrid simulations combining imaging and non-imaging detectors. These simulations are used to explore and optimise the hybrid reconstruction technique and its sensitivity.

The 34th International Cosmic Ray Conference,

30 July- 6 August, 2015

The Hague, The Netherlands

* Speaker. 


\section{Introduction}

Timing arrays like the Hundred*i Square kilometer Cosmic Origin Explorer (HiSCORE)[1] observe the ultra high energy gamma ray and cosmic ray sky by sampling the Cherenkov light of extensive air showers. This technique gives very good reconstruction of core position $(\sim 20 \mathrm{~m})$, energy $(\sim 15 \%)$, and incidence angle $\left(\sim 0.1^{\circ}\right)[5]$ with comparably inexpensive hardware. Since the low fluxes of cosmic rays and gamma rays at and above $100 \mathrm{TeV}$ require huge effective areas (10 $\mathrm{km}^{2}$ and more) and thus make it necessary to instrument large areas, this seems like a promosong way to proceed. The weakness of the timing arrays however is that the gamma-hadron separation power limits the sensitivity, especially at threshold energies $(\sim 10 \mathrm{TeV})$.

Imaging air Cherenkov telescopes, however, achieve a good separation even at the threshold. With two or more telescopes observing the same shower simultaneously, reconstruction of direction and energy are possible as well, but the accuracy deteriorates severely if only one image of a shower is recorded. This sets a limit to the maximum spacing of the telescopes of about $300 \mathrm{~m}$, making very large areas costly.

In the combination of both techniques, the timing array provides the information about shower core position and incidence angle, and the IACT images are used for the identification of the primary particle. This removes the need for IACT stereoscopy and increases the spacing (e.g. $600 \mathrm{~m}$ ), reducing the number of telescopes needed by a factor of four.

As a part of the TAIGA project, the HiSCORE timing array (in deployment) will be combined with small $\left(\sim 10 \mathrm{~m}^{2}\right)$ IACTs (currently in development). HiSCORE stations consist of four large (8") photomultiplier tubes (PMTs), collecting light using Winston cones to sample the shower light front. 28 stations with a spacing of $100 \mathrm{~m}-150 \mathrm{~m}$ covering about $0.25 \mathrm{~km}^{2}$ have so far been deployed in the Tunka valley, Russia ( $51^{\circ} 48^{\prime} 35^{\prime \prime} \mathrm{N}, 103^{\circ} 04^{\prime} 02^{\prime \prime} \mathrm{E}, 675 \mathrm{~m}$ a.s.1.), with the option of increasing the area up to $3 \mathrm{~km}^{2}$.

The IACTs will be of the Davies-Cotton design with a dish diameter of $4.30 \mathrm{~m}$ consisting of 30 circular glass mirror facets with $60 \mathrm{~cm}$ diameter each and a focal length of $4.75 \mathrm{~m}$. The camera consists of 540 PMT pixels with a diameter of the field of view (FoV) of $0.38^{\circ}$ per pixel and $10^{\circ}$ total.

\section{Simulation of IACT}

The Monte Carlo (MC) simulation of the detector performance is done in three steps. First, the shower development is simulated with the CORSIKA-6990[2] code, including the package for EAS Cherenkov light data (IACT option, hadronic interaction models QGSJET [3] and Gheisha [4]). We consider nine telescopes on a regular $3 \times 3$ grid with $600 \mathrm{~m}$ spacing.

Then, each run is processed independently with the HiSCORE and IACT response simulation algorithms.

The HiSCORE response is done by the sim_score[1] code, a custom simulation software based on the IACT package, to determine the resolution for core position, incidence angle, and reconstructed energy. The detection probability for each CORSIKA-generated Cherenkov photon bunch is calculated and applied. For each photon recorded with the detector the response of the electronics 


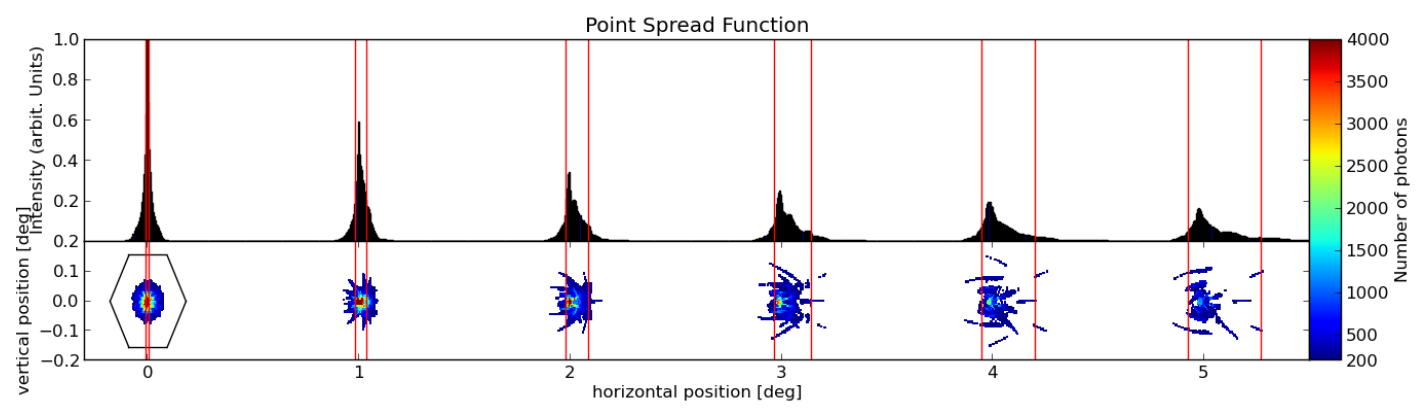

Figure 1: IACT PSF for angles of $0-5^{\circ}$ from the optical axis in 1D projection and in colour scale. Red lines indicate each spot's projected $86 \%$ range. Perfectly aligned mirrors are assumed. The finger-like structures are an effect of the outermost mirror segments.

(with NSB noise and afterpulsing) is done. The simulation lastly performs the same reconstruction routine that is used on the real shower data.

The simulation software for the IACTs is based upon sim_telarray[7]. In sim_telarray, a full raytracing of the Cherenkov photons through the telescopes' optics is performed. Then the electronics response with NSB and electronic noise is generated. Here, all telescope details are included into the simulation, e.g. mirror dish shape, mirror reflectivity and (mis-)alignment, camera size, shadowing by masts and camera, camera PMT efficiency, and the properties of the electronic parts.

sim_telarray also performs image cleaning and the calculation of the Hillas parameters and, if more than one telescope triggered, stereoscopic reconstruction of direction, incidence angle, and energy.

\section{Preliminary Results}

\subsection{IACT Point Spread Function}

Before a combination is possible, one first needs to evaluate if the intended telescope design is adequate. For this, the sim_telarray-given raytracing routine was used to give an estimate on the telescope's point spread function (PSF). The PSF describes how much the image of an infinitly far away point source is widened by the mirror optics.

The Davies-Cotton mirror dish of the TAIGA IACTs is composed of spherical mirror tiles smaller than the overall dish size ( $60 \mathrm{~cm} \varnothing$ on a $4.75 \mathrm{~m} \varnothing$ dish). The tesselation ratio $\alpha$ describes the relation between the diameters of the individual mirror tile $\left(d_{t i l e}\right)$ and the main dish $\left(D_{\text {dish }}\right)$ :

$$
\alpha=\frac{d_{\text {tile }}}{D_{\text {dish }}}
$$

The relative size of the individual mirror tiles is important, with larger tiles the abberation effects due to their spherical nature increase. TAIGA's IACTs are designed with a tesselation ratio of 0.13 .

Figure 1 shows the minimal spread of a point source at different off-axis angles. It can be seen that the PSF is still significantly smaller than a single camera pixel even at an off-axis angle of $5^{\circ}$ at the assumption of perfect mirror alignment. 
TAIGA PSF vs. prediction

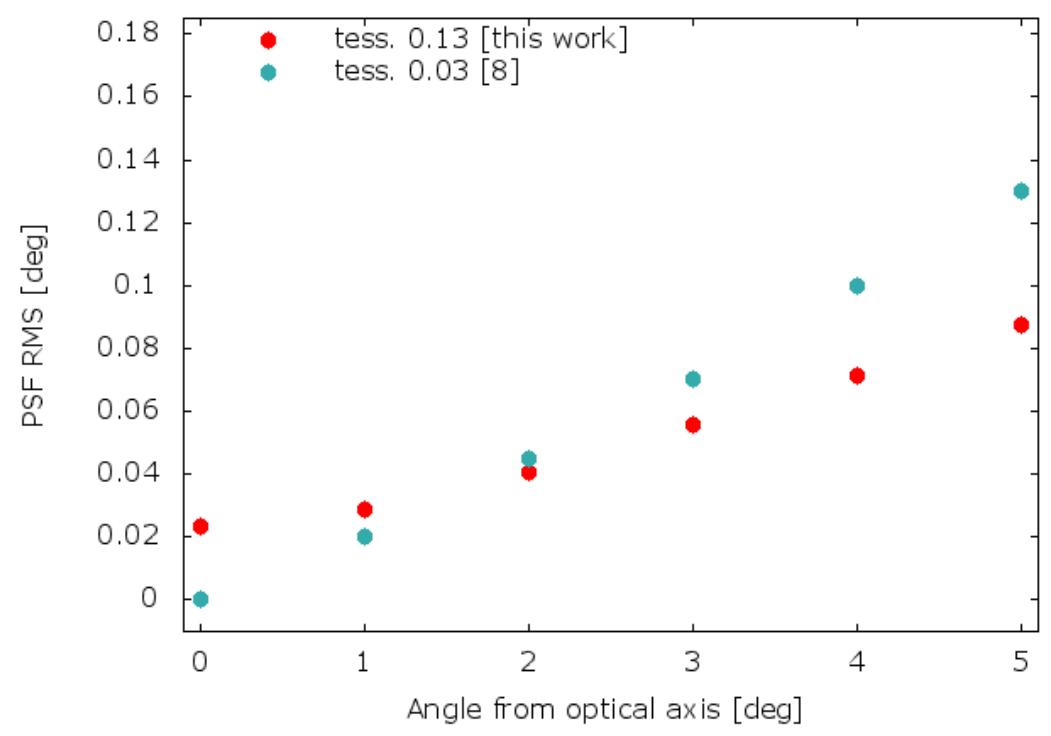

Figure 2: Comparison between analytical and simulated predictions for the PSF for F/D = 1.1. Red: Analytical prediction [8] for a tesselation ratio of 0.03 , Green: Prediction by sim_telarray simulation $(\alpha=0.13)$. The difference in the steepness of the curve is caused by the difference in tesselation ratio. The bigger mirror tiles on the TAIGA IACTs causes the PSF to start larger, but increase less with increasing offset angle.

The impact of the tesselation ratio can be seen in figure 2. While our PSF at small angles is not as good as expected from the analytical predictions by Schliesser and Mirzoyan[8], their assumed tesselation ratio is 0.03 , meaning much smaller mirrors. Smaller mirrors mean less abberation effects from the rims of the tiles, the overall shape gets closer to the shape of the main dish.

\subsection{Gamma-Hadron-Separation using Shower Width}

The main point of the combination between IACT and timing array is to improve the array's gamma-hadron separation power at its sensitivity threshold, e.g. about 10-50 TeV[6]. First, we evaluate how powerful the IACTs are on their own.

The Hillas parameter used for separation is the width of the image, i.e. the second moment of the amplitude distribution after image cleaning. A shower's width is mainly dependant on image size (= overall camera PMT amplitude), impact parameter (= distance between observing telescope and shower core) and nature of the primary. In the combined approach, energy, impact parameter, and direction are measured by the timing array, so the analysis of the IACT images can focus solely on determining if it was a gamma or a hadronic event.

Due to the higher numbers of hadronic interactions in the early stage of a shower, hadron induced showers show a broader width distribution than gamma- or electron-induced showers (see fig.3). A cut on the width can seperate gamma from hadron events. The parameter $Q$ shows the quality of such a cut:

$$
Q=\frac{\varepsilon_{\gamma}}{\sqrt{\varepsilon_{p}}}
$$



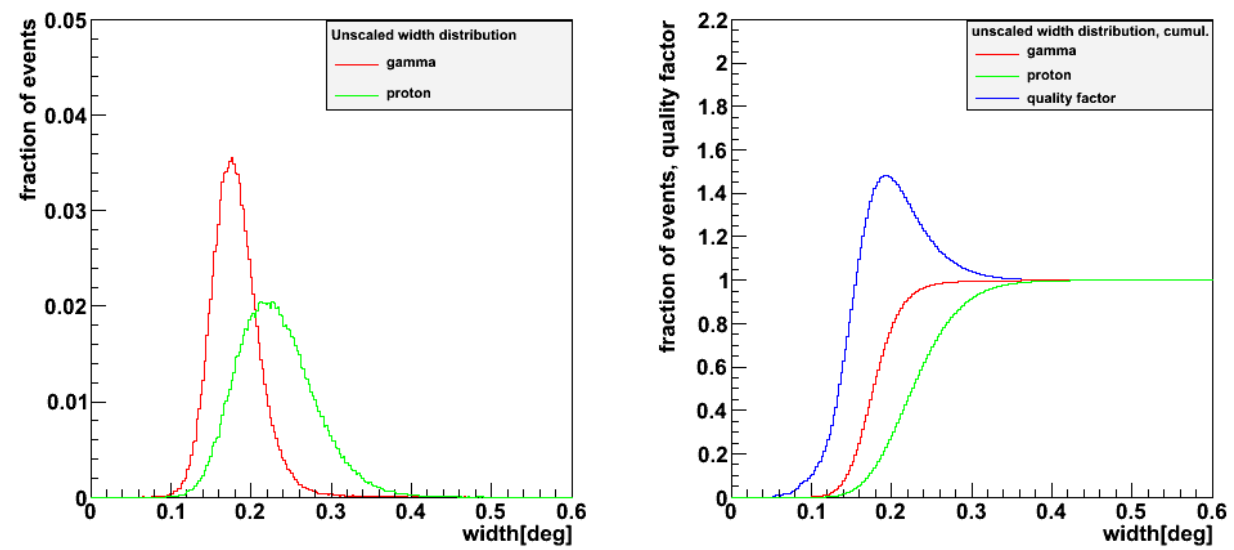

Figure 3: Raw width distribution for showers from the zenith in the energy range of 10-50 TeV. Red: Gammas, Green: Protons. Left: Differential distribution. The proton distribution is broader than the gamma distribution and has a bigger mean, but the two peaks still overlap much. Right: Cumulative distribution and quality factor $\mathrm{Q}$ for the width cut. If a cut is made on the raw data, the maximum quality factor does not reach 1.6.
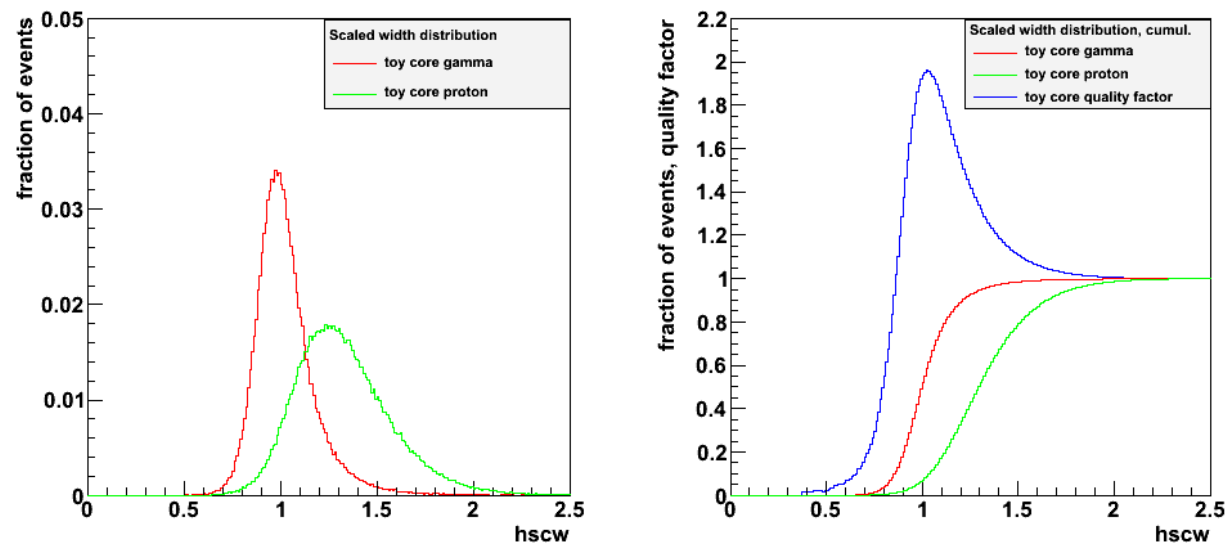

Figure 4: Hybrid scaled width (HSCW) distribution for showers from the zenith in the energy range of 10-50 TeV. Red: Gammas, Green: Protons. Left: Differential distribution. Compared to the unscaled plot, the gamma and the proton distribution have moved apart significantly. Right: Cumulative distribution and quality factor Q for the width cut. If a cut is made on the HSCW, the maximum quality factor is almost 2.

with

$$
\varepsilon_{i}=\frac{n_{i}\left(w<w_{c u t}\right)}{n_{i}}, \varepsilon_{i} \in(\gamma, p)
$$

Natively, the two distributions overlap strongly due to the varying core distances, and a cut on the raw width would yield max. $\mathrm{Q}=1.4$, which is not satisfying. To counter this, the measured widths are rescaled to the expected values of gamma showers, which makes the distributions move apart significantly. For this purpose a lookup-table is generated from MC simulation with the 
dimensions image size after tail cuts ( $\propto$ total image amplitude), impact parameter, zenith angle, and expected width. The individual shower image widths are then divided by their respective lookup table entry, yielding an overall distribution as seen in fig.4. In the actual experiment, this will be the part where the combination takes place: To find the right lookup-table entry for a measured shower, the sampling array is used to find the shower's core distance and zenith angle. The scaled value is called the Hybrid Scaled Width (HSCW):

$$
H S C W=\frac{w_{\text {tel }}}{w_{M C}^{\gamma}\left(d_{\text {array }}, \text { size }_{\text {tel }}\right)}
$$

Cutting on the HSCW instead of on the raw width increases the quality factor of our IACTs from about 1.6 to almost 2, which is better than the separation the HiSCORE array could achieve on its own at these energies. Using the timing array technique alone yields a $\mathrm{Q} \sim 1$ at the threshold and only starts to approach 2 at hundred TeV.[5][6]

For a first estimate on combined reconstruction quality (results shown in fig.4), the true core position is randomised with the HiSCORE core position resolution (toy MC). This randomised postition is then used to find the correct lookup-table entry for scaling, emulating a reconstruction by HiSCORE without actually simulating the showers in sim_score to save computing time.

This lowers the reconstruction quality only marginally as compared to using the 'true' (MC) core position, even though the core resolution for HiSCORE is strongly energy-dependent near the threshold.

\section{Conclusion and Outlook}

The combined reconstruction of HiSCORE and IACTs is of course not restricted to using the core position for a lookup-table. The direction information of the IACTs will be taken into a weighted average with the information that HiSCORE gets from a shower, and the arrival time of the Cherenkov light at a telescope can also be evaluated just like the arrival time on a HiSCORE station. Also, the HiSCORE approach on gamma-hadron information by shower-front profiling will be combined with the image reconstruction. This will probably increase the Q-factor at low energies.

If a shower's impact parameter towards a telescope is too big, the images get truncated at the camera edge which leads to miscalculation of shower axis and width. Typically, these images are discarded by cuts on the Hillas parameter 'distance', but especially for a widely-spaced array like the TAIGA IACTs this case is very common. However, the time gradient inside the shower image can be used to determine the direction of the shower, and the direction of the shower combined with the energy from the array makes it possible to estimate how much of the image has been cut off and how much the true width should be. This could make it possible to discard less events.

The TAIGA system will be the first to combine the strengths of IACT telescopes and Cherenkov timing arrays. The optical quality of the simulated IACT design meets our specifications and the combination between the HiSCORE array and IACTs results in improved gamma-hadron separation and a maximisation of the effective area.

Simulation and reconstruction will be combined to maximise the separation quality and minimise the direction reconstruction uncertainties. 


\section{Acknowledgements}

We acknowledge the support of the Russian Federation Ministry of Education and Science (agreements N 14.B25.31.0010, N2014/15, project 1366, zadanie N 3.889.2014/K), the Russian Foundation for Basic Research (grants 13-02-00214, 15-02-10005, 13-02-12095), The Helmholtz Association (grant HRJRG-303), and the Deutsche Forschungsgemeinschaft(grant TL 51-3)

\section{References}

[1] M. Tluczykont et al, The HiSCORE concept for gamma-ray and cosmic-ray astrophysics beyond 10 TeV Astropart. Phys. 56 42-53 (2014) [1403.5688].

[2] D. Heck et al, Report FZKA 6019 (1998), available from http://www-ik.fzk.de/corsika/physics_description/corsika_phys.html

[3] N. N. Kalmykov, S. S. Ostapchenko, and A. I. Pavlov Nucl. Phys. B (Proc. Suppl.) 52B 17 (1997)

[4] H. Fesefeldt, Report PITHA-85/02 (1985)

[5] D. Hampf, M. Tluczykont and D. Horns, Event reconstruction techniques for the wide-angle air Cherenkov detector HiSCORE Nucl. Inst. Meth. in Phys. Res. A 137-146 (2012/13) [1302.3957]

[6] M. Tluczykont et al, Towards gamma-ray astronomy with timing arrays in proceedings of ECRS (2014)

[7] K. Bernlöhr Simulation of imaging atmospheric Cherenkov telescopes with CORSIKA and sim_telarray, Astropart. Phys. 149-158 (2008)

[8] A. Schliesser and R. Mirzoyan, Wide-field prime-focus imaging atmospheric Cherenkov telescopes: A systematic study, Astrop. Phys.24 382-390 (2005) [astro-ph/0507617] 